\title{
The Pluto Energetic Particle Spectrometer Science Investigation (PEPSSI) on the New Horizons Mission
}

\author{
Ralph L. McNutt Jr. • Stefano A. Livi • Reid S. Gurnee • Matthew E. Hill • \\ Kim A. Cooper • G. Bruce Andrews • Edwin P. Keath • Stamatios M. Krimigis • \\ Donald G. Mitchell • Barry Tossman • Fran Bagenal • John D. Boldt • Walter Bradley • \\ William S. Devereux • George C. Ho - Stephen E. Jaskulek • Thomas W. LeFevere • \\ Horace Malcom • Geoffrey A. Marcus • John R. Hayes • G. Ty Moore • \\ Nikolaos P. Paschalidis • Mark E. Perry • Bruce D. Williams • Paul Wilson IV • \\ Lawrence E. Brown $\cdot$ Martha B. Kusterer $\cdot$ Jon D. Vandegriff
}

Published online: 4 June 2009

(C) Springer Science+Business Media B.V. 2009

Erratum to: Space Sci Rev (2008) 140: 315-385

DOI 10.1007/s11214-008-9436-y

Unfortunately, Nikolaos P. Paschalidis, a co-author of this paper was inadvertently missed from the listing of authors. The complete listing of authors is as given above.

The online version of the original article can be found under doi:10.1007/s11214-008-9436-y.

R.L. McNutt Jr. $(\bowtie) \cdot$ R.S. Gurnee · M.E. Hill · K.A. Cooper · G.B. Andrews · E.P. Keath ·

S.M. Krimigis · D.G. Mitchell · B. Tossman · J.D. Boldt · W. Bradley · W.S. Devereux · G.C. Ho ·

S.E. Jaskulek · T.W. LeFevere - H. Malcom - G.A. Marcus - J.R. Hayes · G.T. Moore · N.P. Paschalidis ·

M.E. Perry · B.D. Williams · P. Wilson IV · L.E. Brown · M.B. Kusterer · J.D. Vandegriff

Applied Physics Laboratory, The Johns Hopkins University, 11100 Johns Hopkins Road, Laurel,

MD 20723, USA

e-mail: Ralph.mcnutt@jhuapl.edu

S.A. Livi

Southwest Research Institute, 6220 Culebra Road, San Antonio, TX 78228, USA

S.M. Krimigis

Academy of Athens, 28 Panapistimiou, 10679 Athens, Greece

F. Bagenal

The University of Colorado, Boulder, CO 80309, USA 